\title{
Wavefront Analysis for Keratoconus
}

\author{
1 Jamyl Habib Castillo, ${ }^{2}$ Rana Hanna, ${ }^{3}$ Eran Berkowitz, ${ }^{4}$ Beatrice Tiosano
}

\begin{abstract}
Keratoconus is the most common primary corneal ectasia, involving a thinning of the central cornea, leading to irregular corneal astigmatism. Wavefront analysis is an evolving field in the area of corneal surgery, initially designed for laser refractive treatments, but today is growing in importance and application in the diagnosis and treatment of corneal disorders such as keratoconus. In this article we will review the theoretical background of high order aberrations and the wavefront analysis as a tool for the diagnosis of keratoconus.
\end{abstract}

Keywords: Keratoconus, Wavefront analysis, High order aberrations, Astigmatism, Cornea, Habib zernike polynomials, Vertical coma.

How to cite this article: Castillo $\mathrm{JH}$, Hanna R, Berkowitz E, Tiosano B. Wavefront Analysis for Keratoconus. Int J Kerat Ect Cor Dis 2014;3(2):76-83.

\section{Source of support: Nil}

\section{Conflict of interest: None}

\section{INTRODUCTION}

Keratoconus is a Greek word (kerato: Cornea; konos: Cone), meaning a cone-shaped protrusion of the cornea. Keratoconus is the most common primary corneal ectasia. It is a noninflammatory, progressive thinning of the cornea that is usually bilateral and involves the central two-thirds of the cornea. The hallmark of this ectatic disorder is an irregular corneal astigmatism. This significant irregularity is the consequence of the changes induced in the anterior corneal geometry. These changes can be assessed by means of corneal topography, demonstrating an increased area of corneal power surrounded by concentric areas of decreasing power, inferior-superior power asymmetry, and skewing of the steepest radial axes above and below the horizontal meridian. ${ }^{1,2}$ These topographic alterations are responsible for the significant induction of higher order aberrations and for visual quality degradation. ${ }^{3}$

\footnotetext{
${ }^{1}$ Attending Doctor, ${ }^{2,3}$ Resident, ${ }^{4}$ Head

${ }^{1-4}$ Department of Ophthalmology, Faculty of Medicine, Hillel Yaffe Medical Center, Israel Institute of Technology, Haifa Hadera, Israel
}

Corresponding Author: Jamyl Habib Castillo, Attending Doctor, Department of Ophthalmology, Faculty of Medicine Hillel Yaffe Medical Center, Israel Institute of Technology, Haifa Hadera, Israel, e-mail: jamylhabib@hotmail.com
Wavefront analysis was initially designed for planning and delivering customized laser refractive treatments but it can also be a valuable diagnostic tool in detecting subtle disorders of the cornea or lens like keratoconus.

\section{Understanding and Measuring Aberrations}

In a 'perfect' optical system, rays emanating from an object point are refracted (and also reflected) to converge to the unique image point expected from Gaussian ray tracing theory. If we consider divergent wavefront rays spreading out from the object to be changed by the optical system into convergent wavefront rays (such as the rays exiting the eye), in a 'prefect' optical media, the wavefronts will be perpendicular to the rays for any phase analyzed (Fig. 1, left). However, if there are aberrations in the optic media, the refracted rays are no longer perfectly converge to the unique image point and are no longer parallel to the rays (Fig. 1, right). Imperfections in the components and materials in the eye may cause light rays to deviate from the desired path. These deviations, referred to as optical or wavefront aberrations, result in blurred images and decreased visual performance. ${ }^{4}$

The wavefront aberrations are measured by the distance between an actual wavefront and an ideal 'perfect' or spherical wavefront (usually in micrometers). This distance represents the difference in the optical path, which is referenced to the center of the 'exit' pupil of the system, where the wavefront aberrations should be zero. Therefore, wavefront aberrations are characterized by the difference between the actual wavefront analyzed and the ideal wavefront measured at the pupil plane. The larger the difference, like in keratoconic corneas, the greater the magnitude of the wavefront aberration error. ${ }^{4}$

Within the past decades, rapid improvement in wavefrontrelated technologies, including the development of sensors for measuring the optical properties of the eye in a clinical environment, allowed the ophthalmic community to move the wavefront theory of light transmission from an academic concept to one being central for better understanding of the effect of aberrations on visual performance and the corresponding image-forming properties of the eye. Imperfections in the optics of the eye are now measured and expressed as wavefront aberration errors. 

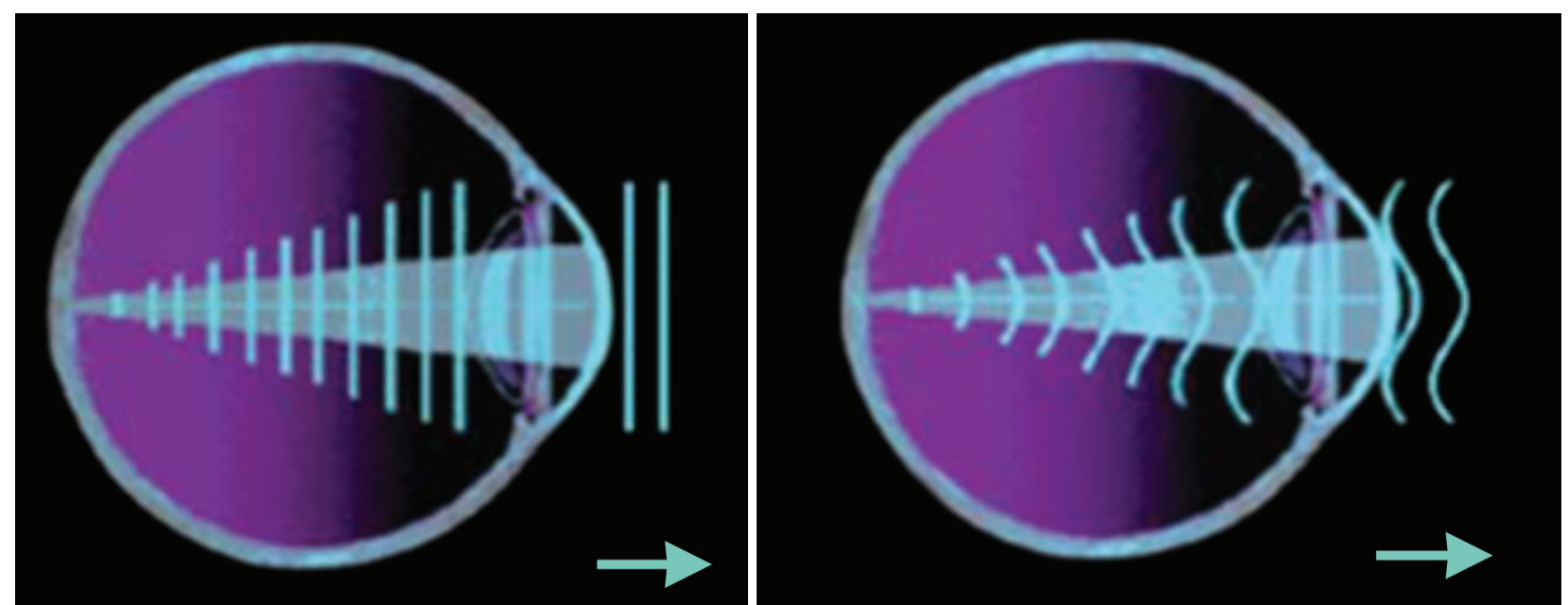

Fig. 1: Left image, planowave front in a perfect optical system. Right image, wavefront aberrations in a representation of a relatively normal eye Images were taken from the textbook on keratoconus: NEW INSIGHTS with permission from the publisher

\section{Zernike Polynomials and Wavefront Aberrations}

Ocular aberrations were classically described in Seidel terms, using functions such as oblique astigmatism, spherical aberration, field curvature and coma. ${ }^{15}$ However, these Seidel terms are limited, in that they cannot describe every single type of aberration found in the eye. As a result this description has been replaced by Zernike's polynomials, ${ }^{16}$ which are complex mathematical formulae that are used to describe the intricate shapes of aberrations across the pupil.

The Zernike polynomials are used in geometry to mathematically describe the best fitting curves.

Each Zernike polynomial coefficient term is arranged and recognized by two features: its angular frequency and its radial order (Formula 1).

$\mathrm{W}(\rho, \theta)=\Sigma \Sigma \mathrm{C} \mathrm{Z}(\rho, \theta)$.

$\mathrm{W}$-Zernike polynomial coefficient

$\rho$ - angular frequency

$\theta$-radial order

Formula 1-The Zernike polynomial coefficient formula.

The coefficient values have both a magnitude and a sign which describes how that particular term makes up a certain proportion of the total wave aberration. The coefficients can tell us the variation in magnitude for each Zernike term used to describe the aberrations. These properties have made Zernike polynomials very attractive in optics. The ordering system for Zernike polynomials starts from 0 (the 0th order) upward with most aberrometers calculating up to the 10th radial order. The values for 'piston' (the 0th radial order coefficient term), 'tip' and 'tilt' (the 1st radial order coefficient terms) are most often ignored when analyzing aberrometry data for normal and abnormal eyes. This is because these terms relate to the displacement of the image only, hence consideration of these with respect to image quality is therefore not usually relevant. ${ }^{17-19}$

Another useful property of Zernike aberrations is that some of the coefficient terms of the Zernike polynomial expansion series are related to known types of optical aberrations such as defocus and astigmatism (2nd order), coma (3rd order) and spherical aberration (4th order).

The most significant optical aberrations are usually manifest in the form of sphere and cylinder, which are known as the lower-order aberration terms of the eye (where $Z(2,0)$ and $Z(2, \pm 2)$ represent sphere and cylinder respectively).

As we go further down the Zernike pyramid (past the 2nd radial order), we move to the components known as the higher order aberrations, the shape of the coefficient term is more complex (Fig. 2).

The amount of aberration is calculated using the root mean square (RMS) of the difference between the actual and the 'ideal' wavefront for every order. The magnitude of the RMS error is dependent on the size of the pupil being investigated, and the amount of wavefront RMS error found will increase as the pupil size increases (Figs $3 \mathrm{~A}$ to $\mathrm{C}$ ). ${ }^{7}$ The wavefront RMS error value allows a brief comparison between different eyes. The higher-order RMS error is the vector sum of all the Zernike terms from the 3rd order and above. The main limitation of the RMS error is that it is only a single value, which does not represent information about the actual shape of the aberrant wavefront itself. Additionally, this value is not directly linked to the retinal image quality of the eye being investigated. ${ }^{20}$

Aberrations are defined by either negative or positive signs as well as a magnitude. A positive sign means that the aberrated wavefront is in front of the ideal plane, whereas a negative sign means that the aberrated wavefront is behind the ideal plane.

\section{Measuring Higher-Order Aberrations}

There are several instruments available today for measuring corneal aberrations (anterior and posterior) and the total ocular aberrations. These include the optical effects of the anterior and posterior cornea as well as the crystalline lens. 


$\begin{array}{llllll} & & & & \\ \end{array}$

Fig. 2: Zernike polynomial pyramid and equations (Images were taken from the textbook on keratoconus: new insights with permission from the publisher)

\section{Types of Wavefront Measuring Systems}

1. Hartmann-Shack aberrometer is an outgoing wavefront aberrometer which is considered the most popular system available in commercial instruments as well as the most common and accepted method of measuring total ocular aberrations in clinical research and in refractive corneal surgery. This system measures the shape of the wavefront reflected out of the eye from a point source on the fovea. An array of microlenslets is used to subdivide the outgoing wavefront into multiple beams which produce spot images on a video sensor (Fig. 3). The displacement of each spot from the corresponding nonaberrated reference position is used to determine the shape of the wavefront. ${ }^{5-9}$

2. Tscherning, or ray-tracing aberrometer is an ingoing instrument. It projects a thin laser beam into the eye, parallel to the visual axis which form a grid, ${ }^{10}$ and the sequential retinal ray tracing of individual points scanning. ${ }^{10-13}$ The scanning of individual points allows the measurement of aberrations in corneas with pronounced optical distortions, which is very useful in cases of keratoconus.

3. Automated retinoscopy, based on dynamic skiascopy. The retina is scanned with a slit-shaped light beam, and the reflected light is captured by an array of rotating photodetectors over a $360^{\circ}$ area. The time difference between projected and reflected light is used to determine the aberrations. ${ }^{14}$

\section{Measuring Corneal Aberrations}

There are several techniques for measuring corneal higherorder aberrations derived from corneal topography data. Briefly these include:

- Placido ring method, e.g. the TMS topographer (Tomey Technology, Waltham, MA, USA)

- Combined slit-scanning and Placido ring technology, e.g. the Orbscan topographer (Bausch and Lomb, Rochester, NY, USA)
- Scheimpflug photography, e.g. the Oculus Pentacam (Wetzlar, Germany)

Of these instruments, the TMS will only measure anterior corneal surface aberrations, whereas the Orbscan II and the Pentacam can measure both anterior and posterior corneal surface aberrations.

\section{Higher-Order Aberrations in Keratoconus}

The amount of higher order aberrations in keratoconic eyes are significantly higher when compared to normal eyes.

In patients with keratoconus, measuring high order aberrations becomes a challenge especially in advanced keratoconus due to the complex corneal irregularity or corneal scars.

When discussing aberrations in keratoconic eyes, vertical come is the most common aberration because corneal thinning is classically located in the inferior temporal part of the cornea. This means that the light waves will distort significantly in different amounts in the superior flatter cornea compared to the inferior steeper cornea, typically showing negative vertical coma aberrations. The cone will also distort incoming light waves by tilting them, including triangular astigmatism or trefoil. Finally the steepened cone also induces spherical aberration. ${ }^{21}$

Several studies have been conducted to prove the sensitivity and specificity of wavefront analysis for the diagnosis of keratoconus based on anterior and posterior corneal aberrations as well as total ocular aberrations. Some of these studies are mentioned below.

\section{Corneal Aberrations Detection using Wavefront Analysis in Patients with Keratoconus}

The detection of keratoconus based on Zernike coefficients from corneal front surface was pioneered by Schwiegerling $^{22,23}$ and has been used in many studies ever since. 

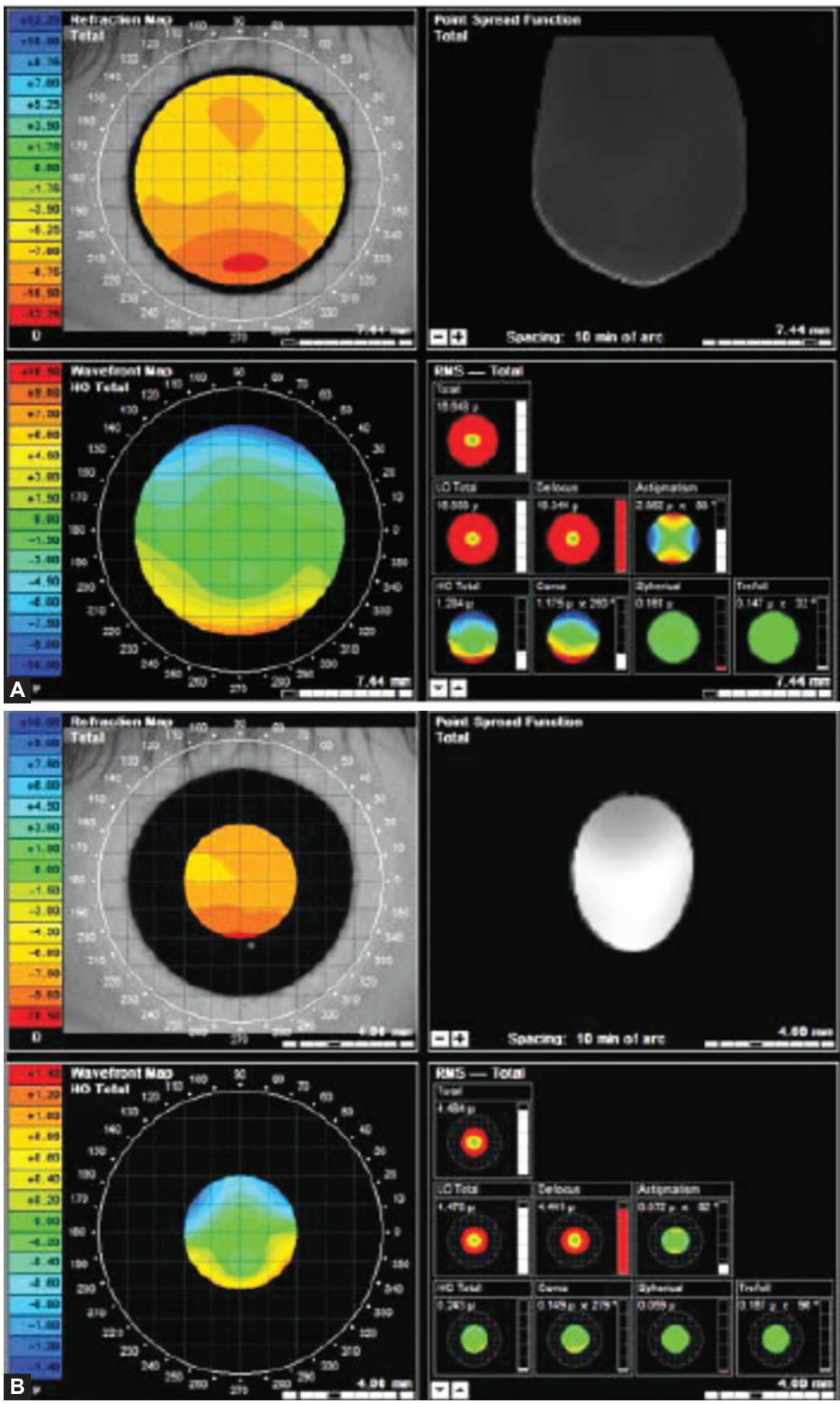

Figs $3 A$ and $B$

Howland et al (1992) ${ }^{24}$ first suggested that measuring and assessing higher-order aberrations derived from corneal topography data may help to distinguish between normal and abnormal corneas. The studies by Schwiegerling et al $(1995)^{25}$ and Schwiegerling and Greivenkamp $(1996)^{23}$ both derived corneal aberrations by expanding their collected data into Zernike terms from videokeratoscopic measurements made using the Placidobased TMS-1 topographer. Schwiegerling and Greivenkamp found elevated absolute values for the 3rd-order Zernike terms coma and trefoil in 15 keratoconic eyes, compared to 61 normal eyes. The data showed that the average keratoconic values for coma and trefoil were significantly different from normal mean values.

Gobbe's $(2005)^{26}$ data for corneal aberrations using the Keraton keratoscope (Optikon, Rome, Italy) and CTView software supported Schwiegerling and Greivenkamp's $(1996)^{23}$ results. They too found elevated amounts of 3rd-order coma and trefoil in keratoconic eyes compared 


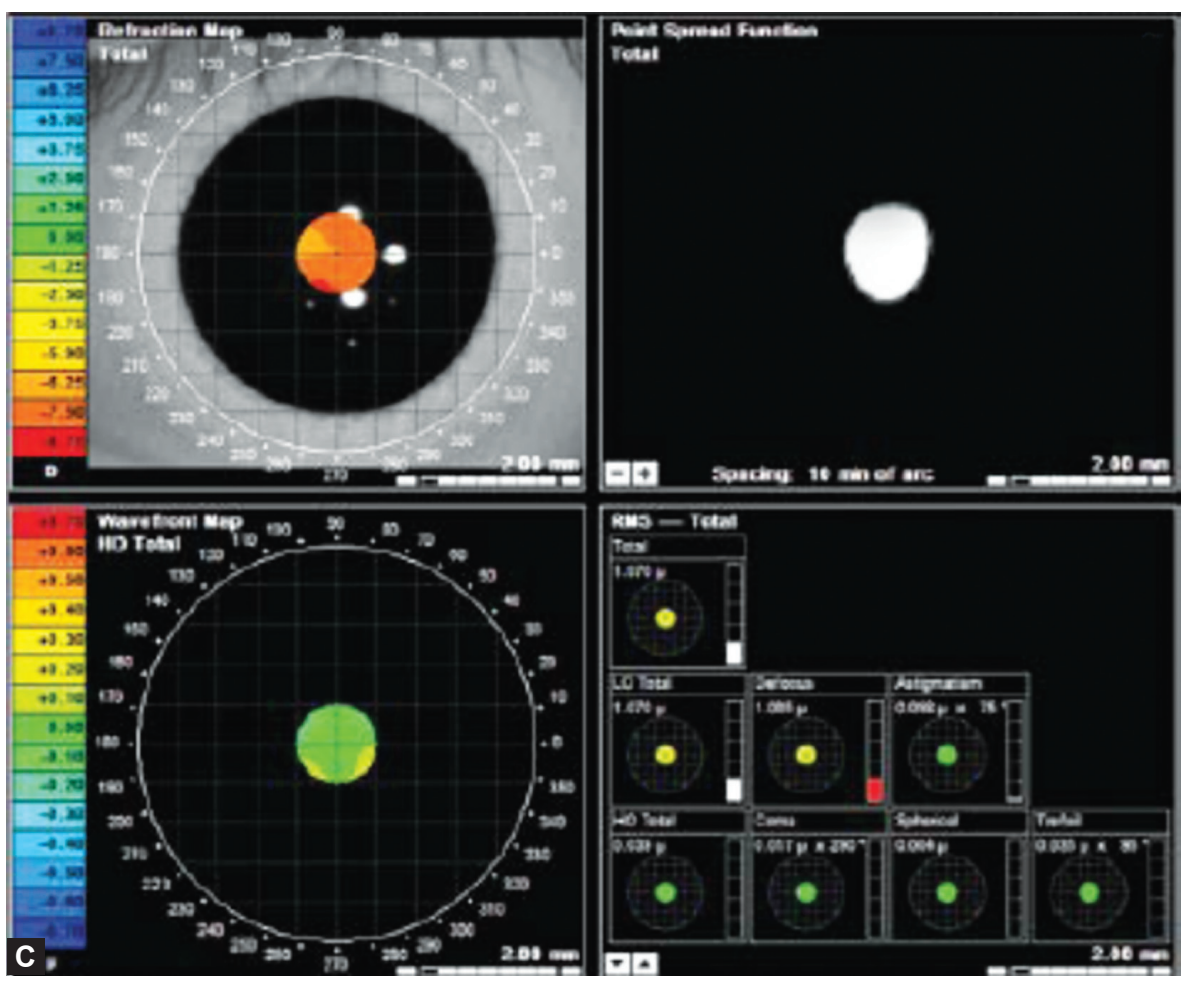

Figs 3A to C: Impact of the scanned pupil area for calculating wavefront aberrations the original scan was $7.44 \mathrm{~mm}$ which enables to calculate the PSF, RMS and all maps up to $2 \mathrm{~mm}$ : (A) Dilated pupil demonstrating higher order aberrations, (B) mild dilated pupil with less aberrations, (C) miotic pupil demonstrating no aberrations at all images were taken from the textbook on keratoconus: new insights with permission from the publisher

to normal eyes, as well as elevated spherical aberrations. The average total 3rd-order RMS value for a $6 \mathrm{~mm}$ corneal aperture was $0.28 \pm 0.15 \mu \mathrm{m}$ in 870 normal eyes $v s 3.10 \pm$ $2.28 \mu \mathrm{m}$ in 73 keratoconic eyes. Their results showed that the best differentiator of keratoconus was negative vertical coma, with a specificity of $71.9 \%$ and a sensitivity of $89.3 \%$. To be deemed 'abnormal' the value of vertical coma should be lower than $-0.12 \mu \mathrm{m}$; to be deemed 'keratoconic' the vertical coma value should be lower than $-0.30 \mu \mathrm{m}$. Gobbe et al were the first to use the aberration values of vertical coma as a diagnostic tool on undiagnosed patients to help detect keratoconus with a high level of sensitivity.

\section{Comparing Corneal Aberrations and Total Ocular Aberrations in Patients with Keratoconus}

Maeda et al (2002) ${ }^{27}$ compared total ocular (from ShackHartmann data) and corneal aberrations (from videokera tographic data) measured using the combined Wavefront Analyzer KR-9000 (Topcon, Tokyo, Japan). Both sets of data were measured and compared between normal and keratoconic eyes. The study found that, compared to normal eyes, keratoconic patients had significantly higher levels of 3 rd-order RMS error in both corneal $(0.26 \mu \mathrm{m}$ in normals $v s$ $1.99 \mu \mathrm{m}$ in keratoconics) and ocular aberration measurements ( $0.25 \mu \mathrm{m}$ in normals $v s 1.83 \mu \mathrm{m}$ in keratoconics). They also found a significant correlation between anterior corneal aberrations and total ocular aberrations for keratoconic eyes, hence suggesting that the anterior surface of the cornea was therefore the major contributor to the total eye aberrations in keratoconus, however the keratoconic eyes investigated ranged from 'suspect' (or forme fruste) to 'mild' keratoconus cases only.

In a later study, Jafri et $\mathrm{al}^{28}$ reported that higher order wavefront aberrations, along with a combination of topography and wavefront variables were effective for distinguishing between early and suspected keratoconus. This prospective comparative study evaluated 50 normal eyes, 10 eyes with early keratoconus, and 10 eyes with suspected keratoconus using Placido's disk-based topography (Tomey TMS-1) and Hartmann-Shack aberrometry (Alcon LADARWave). The RMS for vertical coma, total coma and secondary astigmatism was significantly higher in keratoconus. However, there is an overlap between the groups which limits the ability of total wavefront RMS for the diagnosis of keratoconus. Interestingly, it was the combination of vertical coma and the inferior-superior topographic value that enabled the best distinction between keratoconus and normal eyes.

In another recent study (Sujatha Mohan) ${ }^{29}$ higher-order aberrations were analyzed and compared between 3 groups of patients (Simple refractive errors, high astigmatism, and keratoconus) to determine if they can be used as an 
additional tool for the detection of keratoconus. Corneal topography and analysis of wavefront errors using the Wavelight analyzer II, and Tscherning aberrometry were used in this study. The results of this study demonstrated that Spherical aberrations were significantly higher in the patients with high astigmatism with a mean value of 1.49 when compared with simple refractive errors. Trefoil was also higher in the high cylinder group (mean value 5.579) when compared to the other 2 groups but not statistically significant. Coma and total aberrations were significantly higher in the keratoconus group (mean 0.314 and 2.058 respectively) compared to the other two groups (simple refractive errors and high astigmatism).

Ariela Gordon-Shaag et $\mathrm{al}^{30}$ compared high order aberrations of the cornea and of the eye with inferior-superior (I-S) corneal topography values in keratoconic eyes (including also eyes with suspected keratoconus and eyes without keratoconus). The L80 wave+ instrument was used (it can measure corneal topography and aberrations simultaneously with a large dynamic range) for its ability to evaluate higher order aberrations to the seventh order of the Zernike polynomial function series. The results of this study showed that all ocular and corneal higher aberrations were found to be significantly higher for keratoconic compared to normal eyes, but for suspected keratoconus the results were inconclusive. Corneal aberrations were higher than ocular aberrations due to compensation from the internal aberrations. For manifest keratoconus, the corneal and ocular vertical coma displayed the largest difference (38.6 and 78.5 times higher respectively) compared to normal eyes, while the largest differences for suspected keratoconus were only 5.3 and 4.0 times higher, respectively. In addition, inferiorsuperior dioptric asymmetry was 9.4 and 37.3 times higher for suspected keratoconus and keratoconic eyes respectively, compared to normal eyes. The separation of normality curves between suspected keratocons and normal eyes was $28.6 \%$ for I-S and $14.3 \%$ for both corneal vertical coma and corneal total coma. This comparison highlighted the fact that, traditional corneal topography values such as the I-S dioptric asymmetry remain an important predictor for identifying suspected keratoconus, however, ocular vertical coma and ocular higher order total RMS are a good mean of identifying suspected keratoconus.

\section{Total Ocular Aberrations in Patients with Keratocouns}

Miháltz et $\mathrm{al}^{31}$ in another prospective case-control study evaluated total wavefront aberrations with a HartmannShack sensor on keratoconus and found a significantly higher level of aberrations in Keratoconic eyes compared to normal controls. They also described the changes in the axis of line of sight (LoS) among keratoconic patients. A significant displacement of the LoS was observed in keratoconus and is related to the position of the cone on topography and the vertical coma measured by aberrometry.

\section{Anterior and Posterior Corneal Aberrations in Patients Suffering from Keratoconus}

Jens Buhren ${ }^{32}$ assessed the suitability of corneal anterior and posterior surface aberrations and thickness profile data for discriminating between eyes with early keratoconus (KC), fellow eyes of eyes with early $\mathrm{KC}$ and normal eyes.

In this study, metrics constructed by discriminate analysis obtained from Zernike coefficients from the corneal first surface, detected subclinical KC with excellent accuracy (Vertical coma from the anterior surface was the coefficient with the highest ability to discriminate the group). Adding information from the posterior corneal surface and corneal thickness spatial profiles did not notably increase the discriminative ability, most likely because the correct classification rates utilizing the anterior corneal surface data were already high ('ceiling effect'). In contrast, data obtained from the posterior corneal surface and from corneal thickness spatial profiles alone did not show classification rates superior to those from corneal first-surface data.

Bühren et al (2007), ${ }^{33}$ using the Orbscan II, found significantly elevated levels of vertical coma $(-0.300 \mu \mathrm{m})$, secondary vertical coma $(Z 5,-1)(0.037 \mu \mathrm{m})$ and 3rd-order RMS error $(0.476 \mu \mathrm{m})$, compared to normal control eyes. These results support Gobbe and Guillon's (2005) previous study suggesting that corneal aberration data (as well as corneal height and curvature/shape data) can be useful to help detect 'forme fruste' keratoconus. This study found that for vertical coma the Orbscan recommended a cut-off value of $-0.20 \mu \mathrm{m}$ (or less) in order to be deemed 'abnormal' (or formefruste). Bühren et al showed that mild keratoconic eyes had an average vertical coma value of $-1.35 \mu \mathrm{m}$ compared to $-0.17 \mu \mathrm{m}$ in normal eyes.

David Piñero, Jorge L Alió et al $^{34}$ evaluated the anterior and posterior corneal aberrations provided by the Pentacam system in normal and early to moderate keratoconic eyes.

In this study, two groups were included, one group normal eyes and eyes with the diagnosis of keratoconus. Significant differences were found in all anterior aberrometric parameters except for horizontal primary and secondary coma Zernike terms. Regarding the posterior corneal surface, significant differences among groups were found in primary spherical aberration, primary vertical coma, coma RMS and comalike RMS. In the normal group significantly higher levels of primary vertical coma, spherical aberration, coma and coma-like RMS were found for the posterior corneal surface. 
In the keratoconus group, only significant differences among anterior and posterior corneal surfaces were found in coma RMS, coma-like RMS and vertical coma. These higher levels of aberrations for the posterior corneal surface are in contrast to the theoretical optical properties of this surface. The ratio of refractive indices between air and the anterior surface is $1.0 / 1.376$ and between the aqueous and the posterior surface is $1.376 / 1.336$. This converts to about $1 / 14$ of refraction occurring posteriorly compared to anteriorly.

In conclusion, with Pentacam system, higher levels of vertical coma, primary coma and coma-like aberrations are present in keratoconic eyes compared to normal eyes. The values provided by this device for posterior corneal aberrations were deemed erroneous.

Tomoya et $\mathrm{al}^{35}$ investigated high order aberrations due to the posterior corneal surface in keratoconic eyes compared to normal eyes using a rotating Scheimpflug camera. HOAs (high order aberrations) were calculated at $6 \mathrm{~mm}$ pupils and were expanded with normalized Zernike polynomials. The results demonstrated that the mean total corneal HOAs (RMS) from the anterior-posterior surfaces were significantly higher in keratoconic eyes (4.34/1.09, respectively) compared to the control eyes $(0.46 / 0.15)$. The mean magnitude of each Zernike vector terms for trefoil, coma, and spherical aberrations from the anterior-posterior surfaces was significantly higher in keratoconic $(0.77 / 0.19$, $3.57 / 0.87,-0.44 / 0.17)$ vs control eyes $(0.09 / 0.04,0.33 / 0.07$, $0.25 /-0.07)$, respectively. In conclusion, corneal HOAs on both corneal surfaces in keratoconic eyes were higher than in control eyes. Coma from the posterior surface compensated partly for the anterior surface coma.

\section{SUMMARY}

Corneal front surface aberrations obtained from corneal height data using wavefront technology allow for the detailed description of the optical quality of the cornea. It has been shown to be a reliable tool for the detection of early keratoconus and for the follow-up of the progression of the condition.

Differences between normal corneas and suspected keratoconus were significantly higher in the largest pupil diameters. Hence, differential diagnosis routinely done to detect keratoconic cornea should concentrate on the corneal aberrations measurements in large pupils (a $6.0 \mathrm{~mm}$ diameter pupil has been showed to be useful and is recommended).

When selecting criteria to differentiate between normal and keratoconic (suspected and diagnosed) corneas the overwhelming clinical concern is not to fail in diagnosing an affected patient, hence the test sensitivity is of prime importance. Based on this concern and studies described here, coma along y-axis is the recommended selection criteria. Coma along $y$-axis (Z_13) was the most efficient in detecting both early suspected keratoconus (sensitivity $89.3 \%$, specificity $71.9 \%$ ) and diagnosed keratoconus (sensitivity $94.1 \%$, specificity $88.9 \%$ ). This diagnostic tool could be incorporated into the video keratoscope analysis software and offer a percentage of likelihood whether or not the cornea measured is normal. Assessment of high order aberrations using wavefront analysis can be used as a tool in the diagnosis of keratoconus in addition to other standard methods.

\section{REFERENCES}

1. Maguire LJ, Bourne W. Corneal topography of early keratoconus. Am J Ophthalmol 1989;108:107-112.

2. Klyce SD. Computer-assisted corneal topography. Highresolution graphic presentation and analysis of keratoscopy. Invest Ophthalmol Vis Sci 1984;25:1426-1435.

3. Applegate RA, Hilmantel G, Howland HC, Tu EY, Starck T, Zayac EJ. Corneal first surface optical aberrations and visual performance. J Refract Surg 2000;16:507-514.

4. Ambrósio R Jr, Caldas DL. Diagnosis of keratoconus based on Wavefront Analysis. Textbook on keratoconus: New in Sights Sections 2, Chapter 10, 2012.

5. Thibos LN. Principles of Hartmann-Shack aberrometry. J Refract Surg 2000;16:S563-S565.

6. Charman WN. Wavefront technology: past, present and future. Cont Lens Anterior Eye 2005;28:75-92.

7. Netto MV, Ambrosio R Jr, Schor P, Chalita MR, Chamon W. Wavefront topografia e tomografia da córnea e segmento anterior: atualizaçãopropedêuticaemcirurgiarefrativa. Rio de Janeiro; 2006.

8. McAlinden C, Moore JE. Higher order aberrations using the NIDEK OPD-Scan and AMO WaveScan. J Refract Surg 2014 Aug;26(8):605-608.

9. Cervino A, Hosking SL, Montes-Mico R. Comparison of higher order aberrations measured by NIDEK OPD-Scan dynamic skiascopy and Zeiss WASCA Hartmann. J Refract Surg 2008 Oct;24(8):790-796.

10. Mrochen M, Kaemmerer M, Mierdel P, Krinke HE, Seiler T. Principles of Tscherningaberrometry. J Refract Surg 2000;16: S570-S571.

11. Molebny VV, Panagopoulou SI, Molebny SV, Wakil YS, Pallikaris IG. Principles of ray tracing aberrometry. J Refract Surg 2000;16:S572-S575.

12. Wang L, Wang N, Koch DD. Evaluation of refractive error measurements of the wavescan wavefront system and the tracey wavefront aberrometer. J Cataract Refract Surg 2003;29: 970-979.

13. Wang L, Misra M, Pallikaris IG, Koch DD. Comparison of a ray-tracing refractometer, autorefractor and computerized video keratography in measuring pseudophakic eyes. J Cataract Refract Surg 2002;28:276-282.

14. Gualdi L, Cappello V, Giordano C. The use of NIDEK OPD Scan II wavefront aberrometry in toric intraocular lens implantation. J Refract Surg 2009;25:S110-S115.

15. Atchison and Smith 2000, Born \& Wolf 1999 .

16. Liang J, Grimm B, Goelz S, Bille JF. Objective measurement of wave aberrations of the human eye with the use of a HartmannShack wave-front sensor. J Opt Soc Am A Opt Image Sci Vis 1994;11:1949-1957.

17. Charman WN. Aberrations and myopia. Ophthalmic and Physiological Optics 2005;25:285-301. 
18. Iskander DR. An alternative polynomial representation of the wavefront error function. Invest Ophthalmol Vis Sci 2002;43.

19. Thibos LN, Applegate R. Assessment of optical quality. In MacRae SM, Krueger RR, Applegate RA, editors. Customized Corneal Ablation: The Quest for Super Vision. Thorofare, NJ: Slack 2001; p. 67-78.

20. Marsack JD, Thibos LN, Applegate RA. Metrics of optical quality derived from wave aberrations predict visual performance. $\mathrm{J}$ Vision 2004;4(4):322-328.

21. Jinabhai A. Higher-order aberrations in keratoconus, optometry in practice 2009;10:141-160.

22. Schwiegerling J. Cone dimensions in keratoconus using Zernike polynomials. Optom Vis Sci 1997;74:963-969.

23. Schwiegerling J, Greivenkamp JE. Keratoconus detection based on videokeratoscopic height data. Optom Vis Sci 1996;73: 721-728.

24. Howland HC, Glasser A, Applegate RA. Polynomial approximations of corneal surfaces and corneal curvature topography. Technical Digest Ser Ophthalm Vis Optics OSA 1992;3: 34-37.

25. Schwiegerling J, Greivenkamp JE, Miller JM. Representation of videokeratoscopic height data with Zernike polynomials. J Opt Soc Am (A) 1995;12:2105-2113.

26. Gobbe M, Guillon M. Corneal wavefront aberration measurements to detect keratoconus patients. ContLens Anterior Eye 2005;28:57-66.
27. Maeda N, Fujikado T, Kuroda T, et al. Wavefront aberrations measured with Hartmann-Shack sensor in patients with keratoconus. Ophthalmology 2003 Nov;190(11):1996-2003.

28. Jafri B, Li X, Yang H, Rabinowitz YS. Higher order wavefront aberrations and topography in early and suspected keratoconus. J Refract Surg 2007;23:774-781.

29. Mohan S. Wavefront Analysis as Additional Tool for Detection of Keratoconus.

30. Gordon-Shaag A. Aberrations and topography in normal, keratoconus-suspect, and keratoconic eyes 2012 April;89(4):411-418.

31. Mihaltz K, Kranitz K, Kovacs I, Takacs A, Nemeth J, Nagy ZZ. Shifting of the line of sight in keratoconus measured by a hartmann-shack sensor. Ophthalmology 2010;117:41-48.

32. Buhren J. Detection of subclinical keratoconus by using corneal anterior and posterior surface aberrations and thickness spatial profiles. Investigative Ophthalmology and Visual Science 2010 July 7;51:3424-3432.

33. Buhren J, Kuhne C, Kohnen T. Defining subclinical keratoconus using corneal first-surface higher-order aberrations. Am J Ophthalmol 2007;143:381-389.

34. Piñero D, Alió JL. Pentacam posterior and anterior corneal aberrations in normal and keratoconic eyes. Clin Exp Optom 2009; 92(3):297-303.

35. Nakagawa T. Higher-order aberrations dues to the posterior corneal surface in patients with keratoconus. Invest Ophthalmol Vis Sci 2009 June;50(6):2660-2665. 